\title{
Notes anatomiques sur l'ovaire de quelques Papavéracées
}

\author{
(Suite) ' ;
}

PAR M. 0 . LIGNIER.

\section{Chélidoniées.}

Les Chélidoniées constituent parmi les Papavéracées un type d'autant plus intéressant que le fruit du Chelidonium a été souvent comparé à celui des Crucifères.

Ainsi qu'on le sait, l'ovaire du Macleaya cordata (Willd.) R. $\mathrm{Br}$. est bicarpellé et terminé par deux stigmates presque cylindriques superposés aux carpelles.

Dans cet ovaire encore jeune les deux carpelles sont déjà aplatis (fig. 4, A). Chacun d'eux renferme un très petit faisceau médian, $m$, et, de chaque côté, dans son limbe un réseau vasculaire de cordons très grêles. Dans l'angle de cohérence des carpelles se voit un gros faisceau libéro-ligneux, $p p$, auquel correspond un bourrelet placentaire bien développé. Ce faisceau placentaire est semi-circulaire, et c'est sur ses deux bords que s'insèrent les cordons des quelques ovules distribués en deux lignes. Dans l'ensemble de la section ovarienne ces faisceaux placentaires semblent ètre autonomes non seulement en raison de leur forme, de leur taille et de l'insertion des cordons ovulaires sur leurs deux bords, mais encore parce que de chaque côté d'eux, le parenchyme du limbe ovarien montre un étranglement longitudinal et un début de différenciation qui préparent la rupture ultérieure pour la formation des valves. Cependant de fines commissures transversales ou obliques traversent cette région et relient les faisceaux placentaires au réseau du limbe carpellaire médian (c'est-à-dire des valves).

Dans la base du stigmate la structure externe est presque identique à la précédente (fig. 4, B), sauf, bien entendu, que les bourrelets placentaires y sont papilleux et non ovulifères. Toutefois les faisceaux placentaires commencent a s'y dédoubler longitudinalement en leurs composants. Ce dédoublement se pour-

1. Voir plus haut, p. 279.

T. LVIU.

(SEANCES) 22 
suit en montant dans les stigmates et les quatre composants ainsi libérés se rapprochent des faisceaux médians des carpelles (fig. 4, C et D), pour enfin se fusionner avec
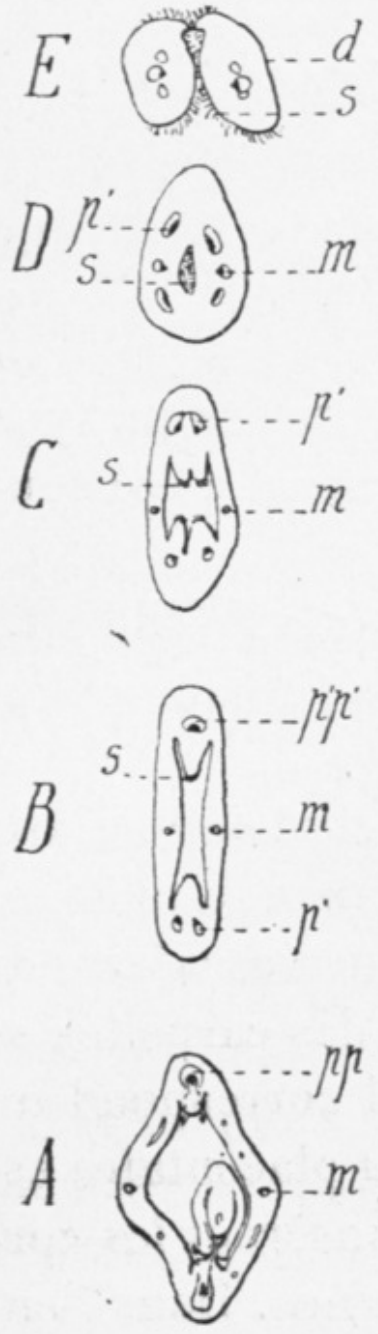

Fig. 4. - Sections transversales d'un ovaire jeune de $\mathbf{M a}$ cleaya cordata: $\mathrm{A}, \mathrm{au}$ milieu de l'ovaire; $\mathrm{B}, \mathrm{C}, \mathrm{D}$, à la base, au milieu et au sommet du style; E, au niveau des stigmates, Gr. 13/2. Mêmes lettres que dans la figure $3 ; p p$, faisceaux marginoplacentaires formés par la fusion des faisceaux marginaux deux à deux. eux dans le sommet des stigmates (fig. 4, E). Simultanément avec ces transformations les bourrelets papilleux se dédoublent également; leurs moitiés s’étalent ensuite en s'étendant vers les plans carpellaires, de façon à recouvrir finalement toute la surface intérieure du style. Plus haut, l'épiderme papilleux recouvre toute la face ventrale des stigmates et s'étend même un peu vers l'extérieur, montrant ainsi que, malgré la forme cylindrique, ces stigmates ont conservé la trace de la réduction dorsale constatée dans ceux des espèces antérieurement décrites.

En résumé, l'ovaire du Macleaya cordata ne renferme que deux carpelles soudés bords à bords et terminés chacun par un stigmate médian. Son organisation générale est semblable à celle des Platystémonées. On pourrait mème dire qu'elle est celle d'un Platystigma dont les placentas seraient très réduits, ou celle d'un Meconella dont les couples placentaires seraient fusionnés en un bourrelet unique, desservi par un faisceau également unique. Toutefois, dans cette dernière comparaison, il existe une différence nette en ce qui concerne le lieu d'insertion des cordons libéro-ligneux ovulaires. Chez le Meconella en effet ils s’insèrent sur les bords externes (les plus rapprochés des marges carpellaires) des faisceaux placentaires encore séparés, tandis que chez le $\boldsymbol{M}$. cordata ils s'insèrent sur leurs bords internes, les seuls que

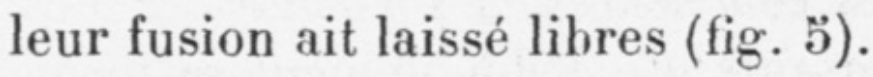

Il m'eut fallu pouvoir comparer également le $M$. cordata avec 
le Romneya qui lui aussi possède des valves. Malheureusement, je l'ai déjà dit, je n'ai pas eu ce genre à ma disposition.

L'ovaire jeune du Chelidonium majus L. et celui du Ch. laciniatum Mill. différent à peine de celui du Macleaya cordata. Ce n'est que tardivement et grâce à des modifications produites par l'accroissement intercalaire qu'ils arrivent à en différer si notablement. Du reste, il suffit de comparer les sections représentées dans la figure 6 avec celles de la figure 4 pour constater cette extrême ressemblance de l'ovaire dans les deux genres. Les seules différences un peu accusées résident dans un moindre aplatissement général chez les Chelidonium et surtout dans le prolongement vers le bas, tout le long de la région ovulifère, $d u$ dédoublement qu'offrent les bourrelets stigmatiques. Encore cette différence ne se fait-elle nullement sentir sur les faisceaux placentaires qui, comme chez le M. cordata, sont ici encore intimement fusionnés en un seul. De telle sorte qu'il est plus juste de dire que l'unique bourrelet placentaire s'est, chez les Chelidonium, dédoublé non pas en raison d'une moindre concrescence des bords des carpelles, mais seulement en raison de la plus grande importance prise par les deux lignes d'insertion des ovules.

Il ne faut donc pas confondre ces couples de bourrelets placentaires des Chelidonium avec ceux déjà signalés chez les Meconella et les Platystemon. Nous avons vu que chez ces derniers les couples résultaient d'une concrescence insuffisante, superficielle des marges placentaires des carpelles. Ici, au contraire, l'accolement des carpelles est très intime puisqu'il va jusqu'à fusionner intimement les faisceaux margino-placentaires en un seul, et le dédoublement des bourrelets est un fait secondaire qui est évidemment en rapport avec le nombre des ovules venant s'ajouter à leur localisation.

On serait peut-être tenté d'admettre qu'il y a reploiement des 
marges carpellaires vers les plans carpellaires, c'est-à-dire que la ligne de coalescence des carpelles a été reportée en arrière de leurs marges jusqu'en face des faisceaux margino-placentaires. Mais c'est là une explication qui, tout d'abord, ne s'accorde pas avec l'organisation des faisceaux placentaires de fusion et qui, d'autre part, sera nettement contre-indiquée dans les genres suivants, tout particulièrement dans le genre Glaucium.
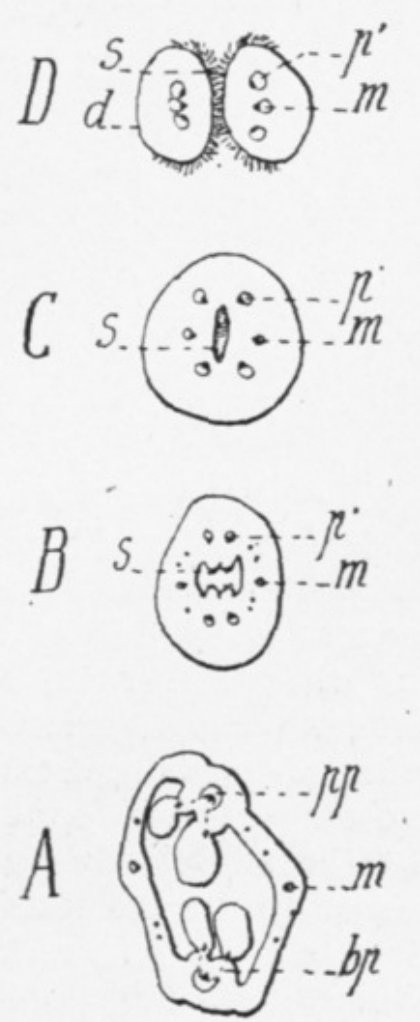

Fig. 6. - Sections transversales d'un ovaire très jeune de Chelidonium lanceolatum: A, au milieu de l'ovaire; B, C, au milieu et au sommet du style; E, au niveau des stigmates, Gr. 13/2. Mêmes lettres que sur les fig. 3 et 4 .

Le Glaucium flavum Crantz est d'ordinaire rangé parmi les Papavérées. Cependant la structure de son ovaire très jeune est trop comparable à celle des Chelidonium pour quil m'ait paru possible de l'en séparer, d'autant plus qu'ellé diffère notablement, par contre, de celle des Papavérées. C'est d'ailleurs à peu près l'opinion à laquelle était arrivé Léger ${ }^{1}$ à la suite de son étude anatomique des organes végétatifs.

Leur ovaire ne comprend que deux carpelles terminés chacun par un stigmate médian, plus aplati et plus élargi à la base que dans les deux genres précédents. Les bords élargis de ces stigmates sont même plus ou moins connés en une sorte de petite languette transversale.

Sur une section transversale de l'ovaire très jeune pratiquée vers sa région médiane, A (fig. 7), les deux limbes carpellaires, pourvus de nombreux faisceaux dont un médian, $m$, sont séparés l'un de l'autre par une région placentaire, $\mathrm{P}$, plus intérieure et, en apparence, absolument autonome. Chacune de celles-ci offre, en effet, l'aspect d'une lame incurvée, sur le dos de laquelle s'insèreraient les bords des limbes carpellaires, et possède un arc de 6 à 9 faisceaux vasculaires dont les plus gros occupent le

1. Léger (L.-J.), Appareil végétatif des Papavéracées (Mém. Soc. Linn. de Normandie, t. XVIII, 1894-95). 
milieu et les plus petits les bords, c'est-à-dire que chacune d'elles a toute l'apparence d'un limbe foliaire indépendant. Pour augmenter l'illusion, ces régions se trouvent normalement en alternance régulière avec les limbes carpellaires médians, de telle sorte qu'en somme elles paraissent représenter un verticille immédiatement plus intérieur. Enfin, entre leur face dorsale et les bords des limbes carpellaires médians, il se produit dans le tissu conjonctif une région spécialisée $r$, chargée de préparer la déhiscence des valves, mais qui a toute l'apparence d'une région de suture. Ajoutons encore que les bourrelets ovulifères, $p b$, sont localisés aux bords de ces lames intérieures et l'on comprendra comment il se fait qu'une telle section de l'ovaire fasse immédiatement songer à l'existence de deux verticilles alternes de deux carpelles chacun, un verticulle extérieur stérile et un verticille intérieur fertile ${ }^{1}$.

Toutefois un examen plus attentif montre $1^{\circ}$ que les bords des arcs libéro-ligneux placentaires sont reliès par de nombreuses commissures gréles, $c$, à ceux des limbes stériles, $2^{\circ}$ que dans ces arcs les faisceaux ont tantôt une disposition impaire, tantòt une disposition paire et qu'ils s'anastomosent entre eux.

Lorsqu'on monte vers le style et les stigmates, les apparences précédentes disparaissent (C, D, E, fig.
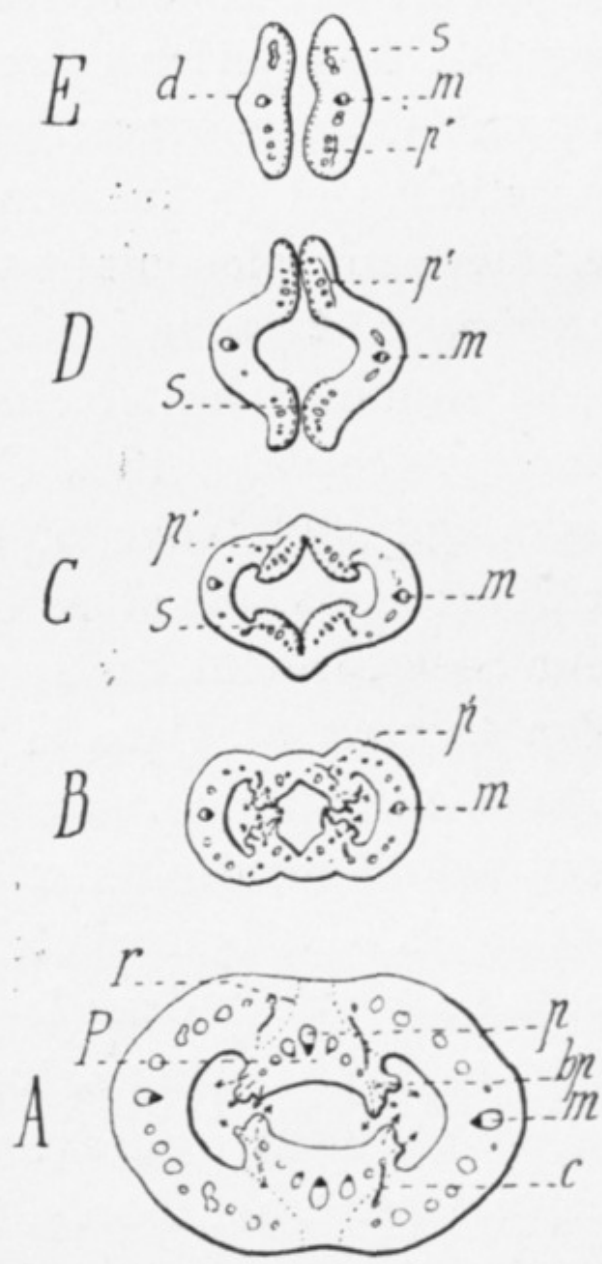

Fig. 7. - Sections transversales de l'ovaire jeune du Glaucium flavum: $\mathbf{A}$, au milieu de la région ovulifère; $B$, au sommet de cette même région; $\mathrm{C}$, au niveau du style; D et E, à la base et au sommet des stigmates, Gr. 13/2. - Mèmes lettres que sur les figures 3 et $4 ; b p$, marge placentaire; $r$, région parenchymateuse dans laquelle se prépare déjà la déhiscence des valves; $c$, commissures libéro-ligneuses qui unissent les bords des arcs placentaires aux bords correspondants des arcs carpellaires.

7). Chaque lame s'y divise en deux $m$ oitiés qui s'écartent lente-

1. La fausse cloison des fruits mûrs manque totalement dans l'ovaire jeune. C'est une formation tardive produite par prolifération des tissus placentaires superficiels. 
ment l'une de l'autre en emportant, chacune, la moitié correspondante du pseudo-arc libéro-ligneux placentaire. Ces moitiés se poursuivent dans la base des stigmates dont elles occupent les bords élargis et un peu épaissis. Là se retrouvent (fig. 7, D) les groupements de faisceaux placentaires, $p^{\prime}$, et les surfaces glandulaires intérieures $s$; plus haut encore, dans le sommet des stigmates, les surfaces glandulaires s'étendent vers les plans carpellaires et s'y fusionnent, $s$ (fig. 7, E), en même temps que les faisceaux placentaires finissent par rejoindre les faisceaux médians $m$ et par se fondre sur leurs bords.

En somme, chez le Glaucium flavum de mème que chez le Macleaya cordata et les Chelidonium, il n'existe que deux carpelles qui sont, chacun, terminés directement par un stigmate médian; mais, en plus, ces stigmates très élargis et connés à leur base forment des sortes de languettes stigmatiques dans le plan transversal. Dans la cavité ovarienne on retrouve les placentas à deux bourrelets ovulifères du Chelidonium, mais ils y sont notablement plus enfoncés vers l'intérieur et beaucoup plus puissants. Ces bourrelets $\mathbf{y}$ sont même séparés par une large lame tangentielle dans laquelle le faisceau unique des Chelidonium est représenté par tout un plexus de cordons libéro-ligneux rangés sur un arc concave vers l'intérieur, les plus gros au milieu, les plus petits aux bords. C'est là un fait qui correspond assurément à un développement considérable, en rapport avec le grand nombre des ovules. De même encore que chez les Chelidonium et le Macleaya cordata, c'est sur les bords internes (les plus rapprochés du plan carpellaire) du faisceau placentaire (en plexus) que s'insèrent les cordons ovulaires; de même aussi les bords de ce aisceau placentaire sont reliés au reste des arcs carpellaires par de nombreuses commissures libéro-ligneuses gréles.

Toutefois, de l'enfoncement des placentas vers l'intérieur et de leur grande extension tranversale, il résulte que l'arc libéroligneux carpellaire subit vers l'intérieur, sur la ligne de jonction de sa partie fertile (placentaire) et de sa partie stérile, un profond plissement longitudinal (fig. 8). Cela donne à la section transversale de l'ovaire un aspect très particulier, et la singularité de cette disposition est encore accentuée par la formation d'un tissu de déhiscence longitudinale des valves qui se produit 
justement à la ligne de plissement. On serait par suite tenté de considérer à tort cette ligne de plissement comme une ligne de suture entre le limbe ovarien extérieur, stérile et le limbe intérieur, fertile.

Ainsi, comme chez le Platystigma lineare et plus même que chez lui, il semble y avoir dans la paroi ovarienne du Glaucium flavum, alternance entre un verticille extérieur formé par deux carpelles stériles et un verticille intérieur constitué par deux carpelles fertiles, ou encore, puisque nous savons que chaque lame placentaire représente deux régions placentaires concrescentes, il semble que chaque carpelle soit trilobé, son lobe médian étant stérile et ses lobes latéraux fertiles sur leur bord interne. Mais la présence des commissures transversales entre ces parties médianes et latérales, de mème que la fusion de leur système vasculaire' dans le sommet du stigmate nous forcent en réalité à reconnaître que ce n'est là qu'une apparence et que les carpelles sont bien réellement entiers. Tout au plus montrent-ils une tendance vers la trilobation, tendance

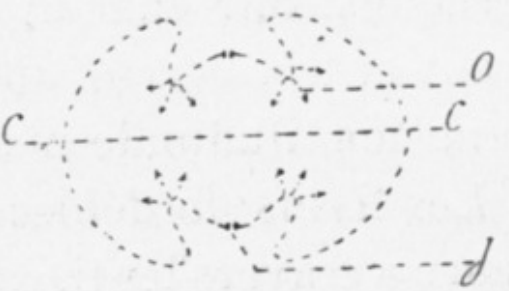

Fig. 8. - Forme schématique des arcs libéro-ligneux carpellaires sur une section transversale de l'ovaire du Glaucium flavum. - CC, plan des carpelles; $n$, plis à la limite des régions fertiles et stériles des arcs carpellaires; $o$, insertion des ovules; $j$, points de jonction des marges carpellaires. qui est peut-être plus affirmée par la forme élargie de la base du stigmate que par le plissement inférieur lui-même.

En résumé, l'ovaire des Chélidoniées ne comprend, comme celui des Platystémonées qu'un seul verticille de carpelles, qui sont coalescents par leurs bords et sont terminés directement par une lamelle stigmatique. Toutefois la coalescence des carpelles y est notablement plus accentuée : elle atteint en effet les faisceaux marginaux qui, se fusionnant deux à deux, forment un unique faisceau placentaire; chez le Gl. flavum celui-ci est remplacé par un plexus de cordons placentaires.

Les ovules qui sont toujours distribués en deux lignes sur chaque placenta, insèrent leurs cordons libéro-ligneux sur les bords de cet unique faisceau (ou du plexus qui le remplace) montrant ainsi une méthode d'insertion différente de celle signalée chez les Platystémonées. 
Chez le Maclaya cordata chaque placenta ne porte qu'un seul bourrelet sur lequel les ovules sont encore insérés en deux lignes. Chez les Chelidonium il porte deux bourrelets côte à côte, séparés seulement par un sillon et donnant insertion aux deux lignes d'ovules. Chez le Glaucium flavum, il existe encore deux bourrelets ovulifères, mais ceux-ci sont très écartés l'un de l'autre, le placenta étant lui-même élargi en une lame tangentielle. En outre grâce à un brusque plissement longitudinal des limbes carpellaires, ces placentas sont ramenés vers l'intérieur et semblent constituer un verticille intérieur alterne avec celui des carpelles. Sur chacun des bourrelets les ovules sont très nombreux et très serrés, aussi ne sont-ils pas répartis sur une simple ligne longitudinale mais sur une bande plus ou moins large.

Les tissus de déhiscence des valves se forment de très bonne heure à travers les tissus du limbe carpellaire. Ils coupent les nombreuses commissures libéro-ligneuses qui unissent les parties valvaires du limbe carpellaire au reste de ce limbe, en particulier aux marges placentaires; ils coupent de même le faisceau médian au sommet des valves.

(A suivre).

\section{Plantes nouvelles, rares ou critiques du bassin moyen du Rhône} (Suite)';

par M. Constant CHASTENiER.

Genre Iris L.

I. lutescens Lamk, Encycl. méth., III, p. 297.

I. olbiensis Hénon in Ann. Soc. agr. hist. nat. et arts ut. Lyon, VIII, p. 463 , t. VI.

Rhizòme plus gros que le doigt. Tige de $15-30 \mathrm{~cm}$., dressée uni-biflore, dépassant plus ou moins longuement les feuilles. Celles-ci larges de 12-25 mm., droites ou arquées. Fleur grande ou très grande (dans un même lieu), pédonculée, à pédoncule plus court que l'ovaire. Feuilles de la spathe ventrues, scarieuses aux bords, obtuses ou subaiguës. Périanthe violet, jaune ou blanchâtre avec des stries et des taches violacées, à tube inclus ou à peine exsert, environ une fois et demie plus 


\section{$2 \mathrm{BHL}$ Biodiversity Heritage Library}

Lignier, Octave. 1911. "Notes anatomiques sur l'ovaire de quelques

Papavéracées (Suite)." Bulletin de la Société botanique de France 58, 337-344. https://doi.org/10.1080/00378941.1911.10832328.

View This Item Online: https://www.biodiversitylibrary.org/item/8680

DOI: https://doi.org/10.1080/00378941.1911.10832328

Permalink: https://www.biodiversitylibrary.org/partpdf/161013

\section{Holding Institution}

Missouri Botanical Garden, Peter H. Raven Library

\section{Sponsored by}

Missouri Botanical Garden

\section{Copyright \& Reuse}

Copyright Status: Public domain. The BHL considers that this work is no longer under copyright protection.

This document was created from content at the Biodiversity Heritage Library, the world's largest open access digital library for biodiversity literature and archives. Visit BHL at https://www.biodiversitylibrary.org. 